\title{
„Das Thema künstliche Intelligenz ist in der Radiologie angekommen“
}

Zum zweiten Mal nach 2017 findet im Februar 2018 der Kongress Emerging Technologies in Medicine - ETIM statt. Initiator des Zukunftskongresses ist Prof. Dr. Michael Forsting, Direktor der Universitätsradiologie Essen. Im Interview skizziert er die Themen der kommenden Tagung und welche Bedeutung sie für die Radiologie haben.

Erneut geht es 2018 um Artificial Intelligence (AI) - was hat sich auf dem Feld in den vergangenen Monaten getan?
Das Thema Al ist in der Radiologie angekommen. Es gibt Start-ups, die gerade nicht aus dem medizinischen Bereich kommen, die aber das Interesse und das Know-how mitbringen, unsere radiologischen Daten zu managen. Die tummeln sich mittlerweile auf radiologischen Kongressen in Amerika, Asien und Europa. Bei Vorträgen zum Thema künstliche Intelligenz sind die Säle voll. Und: Mittlerweile geht es nicht mehr um Spielereien und Experimente, sondern um die Potenziale für die Anwendung. Ein Beispiel aus China: Dort diskutiert man, ob man für die Einführung eines Lungenscreenings auf künstliche Intelligenz setzen soll - das Land hätte gar nicht die erforderliche Zahl an Radiologen, um diese hochspezialisierte Reihenuntersuchung mit menschlicher Hilfe auf die Beine stellen zu können.

Was verbirgt sich hinter dem zweiten Themenschwerpunkt Robotics?

Das Thema Robotics ist primär ein Thema für die Chirurgie, Stichwort assistiertes Operieren. Für die Radiologie sehe ich die Entwicklung auf dem Robotermarkt vor allem im Pflege- und Servicebereich. Radiologie ist ein Fach mit enorm hohem Durchlauf an pflegebedürftigen Patienten, die nicht 


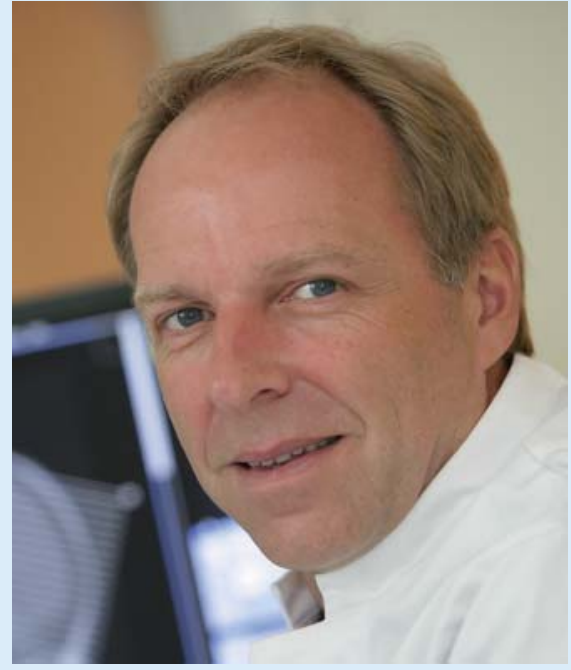

Prof. Dr. Michael Forsting, Tagungsleiter des ETIM 2018 (UK Essen)

nur geröntgt werden müssen, sondern zuvor auch ordnungsgemäß auf dem Untersuchungstisch gelagert. Für diese Arbeit kann ich mir die Unterstützung durch Pflegeroboter sehr gut vorstellen.

Ein Erfolgsrezept des ETIM scheint zu sein, dass nicht nur medizinische Referenten sprechen, sondern auch Entwickler, Ingenieure, Grundlagenforscher...

Die nicht nur im Podium zu finden sind, sondern auch im Publikum! Eine der Aufgaben unseres Kongresses ist es, der Industrie von den Bedürfnissen der Medizin zu erzählen. Ein Unternehmen wie KUKA (führender Hersteller für Roboter und Automatisierungslösungen, die Red.) hat seine primären Märkte in der industriellen Fertigung. Wir wollen denen erzählen, was unsere medizinischen Anforderungen an Robotertechnik sind. Hier müssen Branchen und Denkwelten zusammengebracht werden, die zuvor wenig miteinander zu tun hatten.

Ein Vortrag - es ist der erste Vortrag am Freitagmorgen - greift eine Frage auf, die gerade viel in der radiologischen Community diskutiert wird. Should we be afraid of AI? von Dr. Felix Nensa. Was ist Ihre Meinung: Should we?

Wir sollten Respekt vor der künstlichen Intelligenz haben und bereit sein, die neuen Technologien anzugehen. Al wird uns Radiologen viel Routinearbeit abnehmen können. Ich

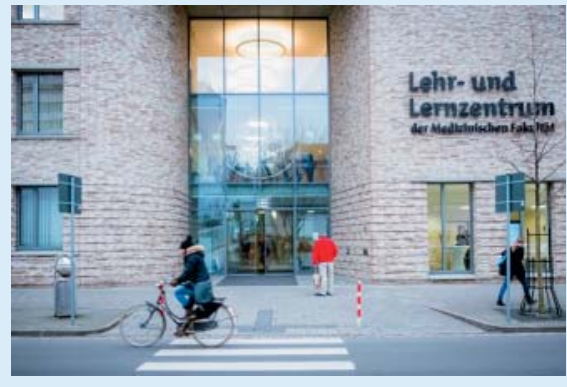

kann mir vorstellen, dass wir in einigen Jahren, sinnvollerweise über das PACS, AI-Applikationen abrufen, die uns sagen, wieviel entzündliche Herde ein MS-Patient hat, Applikationen, die uns beim Tumorstaging helfen oder die Bandscheibenvorfälle auf den Bildern markieren. Das nimmt uns keine
Arbeit weg, es ist ein bisschen wie die Lehrbuchsammlung, die jeder Radiologe heute im Regal stehen hat. Diese Anwendungen entlasten uns, und dies verbessert unsere Medizin. Die Diskussion um den Einsatz von künstlicher Intelligenz darf nicht über Rationalisierungsängste geführt werden, sondern es muss um Qualität gehen. Und wenn künstliche Intelligenz die Qualität der Radiologie verbessert - wovon ich überzeugt bin dann wird sie sich in den Gesundheitssystemen der westlichen Welt durchsetzen.

Quelle: www.dgnr.org 